\title{
Estimating the Performance of Direct-Detection DPSK in Optical Networking Environments Using Eigenfunction Expansion Techniques
}

\author{
João J. O. Pires, Member, IEEE, and Luís G. C. Cancela, Student Member, OSA
}

\begin{abstract}
In-band crosstalk, due to multiple interferers, has been identified as one of the most severe impairments in optical transparent networks, especially in the ones with a large number of nodes and a high wavelength density. Due to its robustness to in-band crosstalk differential phase-shift keying (DPSK) emerges as an attractive modulation scheme to be used in such environments. This paper proposes a rigorous formulation to estimate the performance of direct-detection DPSK receivers using an eigenfunction expansion technique in the time domain. The method takes into account both the in-band crosstalk, due to an arbitrary number of interfering terms, and the amplified spontaneous emission noise and is able to deal with any combination of optical and electrical filter shapes. Using this method the accuracy of an approximation based on the wideband optical filtering assumption was evaluated and shown that the approximation, although not providing reliable results for the error probabilities, can be used with confidence to compute power penalties due to in-band crosstalk. Furthermore, the crosstalk tolerance of DPSK over on-off keying was quantified and shown that this tolerance is reduced when the number of interferers increases.
\end{abstract}

Index Terms-Differential phase-shift keying, eigenvalues and eigenfunctions, in-band crosstalk, optical networking.

\section{INTRODUCTION}

D IFFERENTIAL PHASE-SHIFT KEYING (DPSK) is employed as a modulation scheme in a wide variety of digital communication systems. This scheme entered in the optical arena in the late 1980s, in the context of a research effort that took place at that time in order to use coherent detection techniques as a way to improve receiver sensitivity and hence transmission distance [1], [2]. However, the advent of erbium-doped fiber amplifiers refocused the interest on direct-detection techniques and, as a consequence, the possibility of using a Mach-Zehnder delay interferometer in conjunction with a dual-photodiode balanced receiver, to demodulate optical DPSK signals, started to attract attention [3]-[5]. In the last years, direct-detection DPSK has regained momentum aiming mainly dense wavelength division multiplexing (DWDM) long-haul transmission applications

Manuscript received March 12, 2009; revised December 04, 2009, January 29, 2010; accepted March 03, 2010. Date of publication May 27, 2010; date of current version July $05,2010$.

J. J. O. Pires is with Instituto de Telecomunicações and Department of Electrical and Computer Engineering, Instituto Superior Técnico, 1049-001 Lisboa, Portugal (e-mail: jpires@1x.it.pt).

L. G. C. Cancela is with Instituto de Telecomunicações and Department of Science and Information Technology, Instituto Superior de Ciências do Trabalho e da Empresa, 1649-026 Lisboa, Portugal (e-mail: luis.cancela@ iscte.pt).

Digital Object Identifier 10.1109/JLT.2010.2051319
[6]-[8]. This renewed interest comes from the fact that DPSK outperforms the traditional binary on-off keying (OOK) in such aspects as receiver sensitivity, robustness to transmission impairments, and tolerance to signal power fluctuations [9], [10]. In addition, there is also a significant interest about the promises of this scheme, having in view optical networking applications. Besides the reasons invocated for long-haul transmission, this modulation format presents some properties particularly advantageous in the context of optical networks, including the following: 1) the constant-intensity nature of DPSK favors the use of semiconductor optical amplifier gates in optical switching applications [11], because the patterning effects and the dynamic interactions between DWDM channels due to carrier lifetimes can be greatly reduced [12], [13]; 2) the fact that the decision threshold is zero facilitates the design of optical receivers for burst applications in diverse areas, such as optical packet switching, optical burst switching, and passive optical networking, because it avoids the use of complex decision threshold tracking circuits employed in traditional OOK receivers [14], [15]; 3) the robustness to narrow band optical filtering [16] facilitates the design of transparent optical networks in the measure that contributes to mitigate the cascading filtering effects of optical add-drop multiplexers (OADMs); 4) a higher tolerance to in-band crosstalk than OOK [17] permits to relax the isolation requirements of optical devices, such as multiplexers, and optical switches.

The improved tolerance of DPSK towards crosstalk is particularly advantageous in optical networking environments, because due to imperfections of optical devices used to build network elements, such as OADMs, optical cross-connects, optical burst switches, as well as from spurious reflections inside the network, there are many leakage signals that interfere with the desired signal originating crosstalk [18]-[20]. It is well known that this phenomenon can be particularly damaging when the interference and the signal have the same nominal wavelength, leading to the so-called in-band or homodyne crosstalk, since in this case the signal-crosstalk beatings originated at the receiver can not be filtered out, becoming, as a consequence, an important source of signal quality degradation.

In the context of optical transparent networks its damaging effect is further enhanced due to the fact that the crosstalk accumulates as the signal, corresponding to a given lightpath, traverses multiple network nodes [21]. In networks with a large number of nodes and a high wavelength density this impairment can become one of the most severe sources of performance degradation. Hence, the development of techniques capable of providing accurate performance estimations in the presence of 


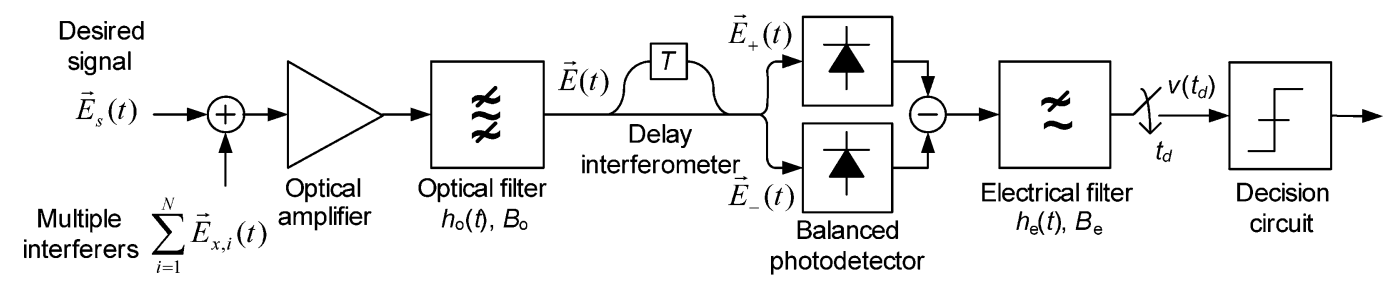

Fig. 1. Schematic diagram of a balanced DPSK optical receiver.

in-band crosstalk is a critically important issue, especially in what concerns the physical layer design of optical transparent networks.

The problem of in-band crosstalk has been the focus of widespread attention in applications based on OOK and a great number of techniques appropriate to evaluate its effect on system performance have been published (see [22] and references therein). These techniques range from simpler ones, based on approximating the statistic of the decision variable as a Gaussian process, to rigorous methods, that rely on the exact statistics. On the contrary, only a few studies have been reported in the literature dealing with the problem of in-band crosstalk in the context of DPSK. In [23], it is derived an analytical expression for the error probability of a DPSK signal considering a single interferer, but the analysis is based on the hypothesis that the optical filter is an ideal matched filter, which is not a viable profile to design optical filters due to current technological constraints. The case of multiple interferers was also studied in this reference using a quite simplistic treatment, based on the Gaussian approximation. A more rigorous and elaborated treatment to analyze the performance of DPSK receivers in the presence of multiple interferers is described in [24]. However, this treatment is based on the assumption that the number of interferers is infinite, which is not a sufficiently general assumption to accurately describe many of the circumstances encountered in real optical networks. Another published approach was proposed by the authors to estimate the bit-error rate of DPSK systems in the presence of amplified spontaneous emission (ASE) noise and in-band crosstalk due to a single [25], and multiple interfering signals [26]. Nevertheless, this analysis relies on a simplified assumption, since it considers an idealized receiver resulting from the combination of an ideal rectangular optical filter with large bandwidth-time product and an integrate-and-dump electrical filter, the so-called wideband optical filtering assumption [27], [28]. It is also worth noting that due to the complex nature of the statistics of the decision variable, the Gaussian techniques can not be applied to give accurate results with DPSK signals, even when the number of interferers is high [29], restricting considerably the space of solutions in comparison with OOK. In our recent work we have also developed some theory to deal with coherent in-band crosstalk originated from multipath interference, but once again its foundation relies on the wideband assumption [30].

In the present paper, we provide a general formulation for estimating the performance of optically pre-amplified DPSK receivers in the presence of in-band crosstalk due to an arbitrary number of interferers, by extending our previous work to the case of arbitrary optical and electrical filtering. The formulation uses an eigenfunction expansion technique to decompose the signal, the interference, and the ASE noise, at the optical filter input, in terms of a series of orthogonal functions and relies on the moment generating function (MGF) to describe the statistics of the decision variable. This approach follows the treatment introduced by Emerson to study square law detectors [31] and has some similarities with the rigorous approaches reported in [10], and [32] to evaluate the bit-error rate of DPSK receivers. The main difference is that [10] uses the eigenfunction expansion in the frequency domain, rather than in the time domain, as happens here, whereas [32] uses an expansion at the optical filter output, leading to a different integral equation. In comparison with [32], our approach has the advantage of simplifying the orthonormality conditions, as well as the computation of the expansion coefficients, since they become independent of the electrical filter impulse response. It may be noted that the Forestieri method [33] has also been applied in rigorous studies in the context of direct-detection DPSK receivers [9], [34]. However, this method uses the Fourier series as the orthornormal basis for performing expansions, which differs from our strategy of computing a basis function for each combination of optical and electrical filters.

The remainder of this paper is structured as follows. In Section II, we describe the model used to characterize the decision variable at the DPSK receiver output, and show how this variable can be written as a series of independent random variables in order to deal with arbitrary filtering. In Section III, the MGF of the decision variable is derived and the application of the saddle-point integration method to error probability and probability density evaluation is discussed. In addition, the simplifications subjacent to the wideband optical filtering assumptions are also outlined. Numerical results are given in Section IV and some concluding remarks are provided in Section V.

\section{Decision VARIABle Modeling AND EIGENFUNCTION EXPANSION}

The structure of a typical direct-detection DPSK receiver using balanced detection is shown in Fig. 1. It consists of an optical pre-amplifier with gain $G$, an optical filter, a delay interferometer with a differential delay equal to the bit period $T$, a balanced photodetector, and a post-detection electrical filter. The optical filter is assumed to have an arbitrary low-pass equivalent impulse response $h_{o}(t)$ and an optical bandwidth $B_{o}$, whereas the electrical filter is described by the impulse response $h_{e}(t)$ and by the electrical bandwidth $B_{e}$.

In a DPSK signal the binary information is transmitted by shifting the phase of the optical carrier, by either 0 or $\pi$, between adjacent signaling intervals. Therefore, the delay interferometer acts as an optical demodulator, converting the phase modulation 
to intensity modulation. Here, we assume that when the symbol "one" is received the signal emerges from the constructive port, and when the symbol "zero" is received it emerges from the destructive port. Suppose that at the receiver the incoming DPSK signal is impaired by in-band crosstalk due to $N$ DPSK interferers, originated from $N$ different sources with the same bit rate and nominal wavelength as the desired signal. Therefore, the total electrical field at the interferometer input can be given by

$$
\vec{E}(t)=\vec{E}_{s}(t)+\sum_{i=1}^{N} \vec{E}_{x, i}(t)+\vec{E}_{n}(t)
$$

where the first term corresponds to the desired signal, the second term to the in-band crosstalk, and the third term to the ASE noise originated from the optical pre-amplifier. The complex envelope of the signal field during the interval $[0, T]$ can be represented as

$$
\vec{E}_{s}(t)=\left\{\sqrt{2 G P_{s}} u(t) \exp \left[j \theta_{s}(t)\right] \vec{r}\right\} * h_{o}(t)
$$

where $*$ denotes convolution, $P_{s}$ the average signal power incident at the amplifier input, $u(t)$ a rectangular pulse of unitary amplitude within the interval $[0, T]$ and zero elsewhere, $\vec{r}$ the polarization unit vector, and $\theta_{\mathrm{S}}(t)$ the signal phase. This phase is given by $\theta_{s}(t)=\theta_{s}(t-T)+\pi\left(1-a_{s}\right) / 2$, where $\theta_{s}(t-T)$ is the phase in the previous time interval, and $a_{s}=1$ for symbol "one" and $a_{s}=-1$ for the symbol "zero". The complex envelope of the $i$-th interfering signal in turn can be given by

$$
\vec{E}_{x, i}(t)=\left\{\sqrt{2 G P_{x, i}} u(t) \exp \left[j \theta_{x, i}(t)+j \phi_{x, i}\right] \vec{r}\right\} * h_{o}(t)
$$

where $P_{x, i}$ is the average crosstalk power at the amplifier input, $\theta_{x, i}(t)=\theta_{x, i}(t-T)+\pi\left(1-a_{x, i}\right) / 2$ is the phase conveying the information, with $a_{x, i}=1$ for the symbol "one" and $a_{x, i}=$ -1 for the symbol "zero", and $\phi_{x, i}$ is a random phase. The crosstalk level of the $i$-th interferer $\varepsilon_{i}$ is defined as the ratio between the crosstalk power and the signal power $\left(\varepsilon_{i}=P_{x, i} / P_{s}\right)$, whereas the total crosstalk level is given by $\varepsilon_{T}=\sum_{i=1}^{N} \varepsilon_{i}$. Throughout this paper it is considered a worst case interference scenario reflected in the fact that all the interfering signals are assumed to be co-polarized and temporally aligned with the desired signal. In practice, the polarization vectors are random, but as they show a statistical tendency to be aligned and stay in this state for a long time [35], the co-polarization scenario is justified. The ASE noise due to the pre-amplifier is considered to be a zero mean white stationary Gaussian noise with a single-sided power spectral density in each polarization given by $N_{o}=h \nu_{s}(G-1) F / 2$, where $h \nu_{s}$ is the photon energy at the signal wavelength, and $F$ is the noise factor. We assume that the gain $G$ is sufficiently high, so that the ASE noise dominates over shot noise and thermal noise in the receiver, allowing us to neglect these noises in the present analysis. The field $\vec{E}_{n}(t)$ can be expressed in terms of in-phase $n_{c}(t)$, and quadrature $n_{s}(t)$ components, giving for the case of the ASE noise having the same polarization as the signal $\vec{E}_{n}(t)=\left\{\left[n_{c}(t)+j n_{s}(t)\right] \vec{r}\right\}_{*} * h_{o}(t)$. The electrical fields at the interferometer outputs are $\vec{E}_{+}(t)=$ $(1 / 2)[\vec{E}(t)+\vec{E}(t-T)]$ for the constructive port and $\vec{E}_{-}(t)=$ $(1 / 2)[\vec{E}(t)-\vec{E}(t-T)]$ for the destructive port. These fields are detected using a pair of identical photodiodes with unitary responsivities and the resulting currents are subtracted and filtered by the electrical filter. The decision variable $v$ at the filter output, defined at the decision time $t_{d}$, can then be written as

$$
\begin{aligned}
v\left(t_{d}\right)= & v^{+}\left(t_{d}\right)-v^{-}\left(t_{d}\right) \\
= & \int_{-\infty}^{+\infty} i_{+}\left(t_{d}-\varsigma\right) h_{e}(\varsigma) d \varsigma \\
& -\int_{-\infty}^{+\infty} i_{-}\left(t_{d}-\varsigma\right) h_{e}(\varsigma) d \varsigma
\end{aligned}
$$

where $i_{+}(t)=\left|\vec{E}_{+}(t)\right|^{2} / 2$, and $i_{-}(t)=\left|\vec{E}_{-}(t)\right|^{2} / 2$. The decision variable $v$ is the difference between the random variable $v^{+}\left(t_{d}\right)$, resulting from the constructive port, and the random variable $v^{-}\left(t_{d}\right)$, resulting from the destructive port. These random variables can be rewritten as

$$
\begin{aligned}
v^{+}\left(t_{d}\right)= & \frac{1}{2} \int_{-\infty}^{+\infty} \int_{-\infty}^{+\infty} \Lambda\left(\tau_{1}, \tau_{2}\right) \\
& \times\left\{\left[y_{1 c}\left(t_{d}-\tau_{1}\right)+n_{1}\left(t_{d}-\tau_{1}\right)\right]\right. \\
& \times\left[y_{1 c}\left(t_{d}-\tau_{2}\right)+n_{1}\left(t_{d}-\tau_{2}\right)\right] \\
& +\left[y_{1 s}\left(t_{d}-\tau_{1}\right)+n_{2}\left(t_{d}-\tau_{1}\right)\right] \\
& \left.\times\left[y_{1 s}\left(t_{d}-\tau_{2}\right)+n_{2}\left(t_{d}-\tau_{2}\right)\right]\right\} d \tau_{1} d \tau_{2}
\end{aligned}
$$

and

$$
\begin{aligned}
v^{-}\left(t_{d}\right)= & \frac{1}{2} \int_{-\infty}^{+\infty} \int_{-\infty}^{+\infty} \Lambda\left(\tau_{1}, \tau_{2}\right) \\
& \times\left\{\left[y_{2 c}\left(t_{d}-\tau_{1}\right)+n_{3}\left(t_{d}-\tau_{1}\right)\right]\right. \\
& \times\left[y_{2 c}\left(t_{d}-\tau_{2}\right)+n_{3}\left(t_{d}-\tau_{2}\right)\right] \\
& +\left[y_{2 s}\left(t_{d}-\tau_{1}\right)+n_{4}\left(t_{d}-\tau_{1}\right)\right] \\
& \left.\times\left[y_{2 s}\left(t_{d}-\tau_{2}\right)+n_{4}\left(t_{d}-\tau_{2}\right)\right]\right\} d \tau_{1} d \tau_{2}
\end{aligned}
$$

where $\Lambda\left(\tau_{1}, \tau_{2}\right)$ is defined by

$$
\Lambda\left(\tau_{1}, \tau_{2}\right)=\int_{-\infty}^{+\infty} h_{e}(\varsigma) h_{o}\left(\tau_{1}-\varsigma\right) h_{o}\left(\tau_{2}-\varsigma\right) d \varsigma
$$

and

$$
\begin{aligned}
y_{1 c, 2 c}(t)= & \sqrt{2 G P_{s} \alpha_{s}^{ \pm}} u(t) \\
& +\sum_{i=1}^{N} \sqrt{2 G P_{x, i} \alpha_{x, i}^{ \pm}} u(t) \cos \Delta \theta_{i}(t) \\
y_{1 s, 2 s}(t)= & \sum_{i=1}^{N} \sqrt{2 G P_{x, i} \alpha_{x, i}^{ \pm}} u(t) \sin \Delta \theta_{i}(t) \\
n_{1}(t)= & (1 / 2)\left[n_{c}(t)+n_{c}(t-T)\right] \\
n_{2}(t)= & (1 / 2)\left[n_{s}(t)+n_{s}(t-T)\right] \\
n_{3}(t)= & (1 / 2)\left[n_{c}(t)-n_{c}(t-T)\right] \\
n_{4}(t)= & (1 / 2)\left[n_{s}(t)-n_{s}(t-T)\right] .
\end{aligned}
$$

In the above equations $\Delta \theta_{i}(t)=\theta_{x, i}(t-T)-\theta_{s}(t-T)+$ $\phi_{x, i}, \alpha_{s}^{ \pm}=\left(1 \pm a_{s}\right)^{2} / 4$, and $\alpha_{x, i}^{ \pm}=\left(1 \pm a_{x, i}\right)^{2} / 4$. The random process $\Delta \theta_{i}(t)$ represents the difference between the accumulated phases of the $i$-th interferer and the signal, and includes also the phase $\phi_{x, i}$. This phase can be viewed as the relative phase between the signal and the $i$-th interferer and depends, namely, on the laser phase noise [23]. Since the laser coherence time typically exceeds the bit period $T$, the phase $\phi_{x, i}$ can be assumed constant over this period and uniformly distributed in $[0,2 \pi]$. Bearing in mind the properties of uniformly distributed 
random processes [36] the process $\Delta \theta_{i}(t)$ is also modeled as uniform over the same interval. It is also reasonable to assume that all the $\Delta \theta_{i}^{\prime} s$ are statistically independent, because the $N$ interferers are originated from different sources.

The function $\Lambda\left(\tau_{1}, \tau_{2}\right)$ is continuous and is also symmetric because $\Lambda\left(\tau_{1}, \tau_{2}\right)=\Lambda\left(\tau_{2}, \tau_{1}\right)$. Furthermore, if the condition $h_{e}(t) \geq 0$ is satisfied this function is in addition positive semidefinite and the Mercer's theorem can be applied giving [31]

$$
\Lambda\left(\tau_{1}, \tau_{2}\right)=\sum_{k=0}^{\infty} \lambda_{k} \varphi_{k}\left(\tau_{1}\right) \varphi_{k}\left(\tau_{2}\right)
$$

where $\varphi_{k}(t)$ is the $k$ th eigenfunction, and $\lambda_{k}$ the corresponding eigenvalue of the integral equation

$$
\int_{-\infty}^{+\infty} \Lambda(t, \tau) \varphi_{k}(\tau) d \tau=\lambda_{k} \varphi_{k}(t)
$$

and, as a result, the functions $\left\{\varphi_{k}(t)\right\}$ satisfies the orthonormality condition

$$
\int_{-\infty}^{+\infty} \varphi_{j}(t) \varphi_{k}^{*}(t) d t=\delta_{j k}
$$

where $\delta_{j k}$ is the Kronecker delta function. Inserting (10) into (5) and (6) we get after some algebra

$$
\begin{aligned}
& v^{+}=\frac{1}{2} \sum_{k=0}^{\infty} \lambda_{k}\left[\left(y_{1 c, k}+n_{1, k}\right)^{2}+\left(y_{1 s, k}+n_{2, k}\right)^{2}\right] \\
& v^{-}=\frac{1}{2} \sum_{k=0}^{\infty} \lambda_{k}\left[\left(y_{2 c, k}+n_{3, k}\right)^{2}+\left(y_{2 s, k}+n_{4, k}\right)^{2}\right]
\end{aligned}
$$

with

$$
\begin{aligned}
y_{1 c, k}= & \sqrt{2 G P_{s} \alpha_{s}^{+}} \int_{-\infty}^{+\infty} u\left(t_{d}-\tau\right) \varphi_{k}(\tau) d \tau \\
& +\sum_{i=1}^{N} \sqrt{2 G P_{x, i} \alpha_{x, i}^{+}} \int_{-\infty}^{+\infty} u\left(t_{d}-\tau\right) \\
& \times \cos \Delta \theta_{i}\left(t_{d}-\tau\right) \varphi_{k}(\tau) d \tau \\
y_{1 s, k}= & \sum_{i=1}^{N} \sqrt{2 G P_{x, i} \alpha_{x, i}^{+}} \int_{-\infty}^{+\infty} u\left(t_{d}-\tau\right) \\
& \times \sin \Delta \theta_{i}\left(t_{d}-\tau\right) \varphi_{k}(\tau) d \tau
\end{aligned}
$$

and

$$
n_{l, k}=\int_{-\infty}^{+\infty} n_{l}\left(t_{d}-\tau\right) \varphi_{k}(\tau) d \tau, \quad \text { for } l=1, \ldots, 4
$$

where $y_{2 c, k}$ is given by (15) with $\alpha_{s}^{+}$replaced by $\alpha_{s}^{-}$and $\alpha_{x, i}^{+}$ replaced by $\alpha_{x, i}^{-}$, whereas $y_{2 s, k}$ is given by (16) with $\alpha_{x, i}^{+}$replaced by $\alpha_{x, i}^{-}$. In (17), the random coefficients $\left\{n_{l, k}\right\}$ are mutually independent zero-mean Gaussian random variables, with variances $\left\langle n_{l, k}^{2}\right\rangle=N_{0} / 2$, for $l \in\{1, \ldots, 4\}$, since according with (17) and (12) we have

$$
\left\langle n_{l, j} n_{l, k}\right\rangle=\int_{-\infty}^{+\infty} \int_{-\infty}^{+\infty}\left\langle n_{l}\left(t_{d}-\tau_{1}\right) n_{l}\left(t_{d}-\tau_{2}\right)\right\rangle
$$

$$
\begin{aligned}
& \quad \times \varphi_{j}\left(\tau_{1}\right) \varphi_{k}\left(\tau_{2}\right) d \tau_{1} d \tau_{2} \\
& =\frac{1}{2} \int_{-\infty}^{+\infty} \int_{-\infty}^{+\infty} N_{0} \delta_{d}\left(\tau_{1}-\tau_{2}\right) \\
& \quad \times \varphi_{j}\left(\tau_{1}\right) \varphi_{k}\left(\tau_{2}\right) d \tau_{1} d \tau_{2}=\frac{1}{2} N_{0} \delta_{j k}
\end{aligned}
$$

where $\delta_{d}(t)$ is the Dirac delta function and the operator $\langle\cdot\rangle$ denotes the expected value. An additional simplification can be obtained in (15) and (16) by assuming that $\Delta \theta_{i}$ is constant over the interval $[0, T]$ giving

$$
\begin{aligned}
& y_{1 c, k}=\sqrt{2 G P_{s} \alpha_{s}^{+}} u_{k}+\sum_{i=1}^{N} \sqrt{2 G P_{x, i} \alpha_{x, i}^{+}} u_{k} \cos \Delta \theta_{i} \\
& y_{1 s, k}=\sum_{i=1}^{N} \sqrt{2 G P_{x, i} \alpha_{x, i}^{+}} u_{k} \sin \Delta \theta_{i}
\end{aligned}
$$

where

$$
u_{k}=\int_{-\infty}^{+\infty} u\left(t_{d}-\tau\right) \varphi_{k}(\tau) d \tau=\int_{-T+t_{d}}^{t_{d}} \varphi_{k}(\tau) d \tau .
$$

If the ASE noise in the polarization orthogonal to the signal is taken into account, then (13) and (14) must be written in the following form:

$$
\begin{aligned}
v^{+}= & \frac{1}{2} \sum_{k=0}^{\infty} \lambda_{k}\left[\left(y_{1 c, k}+n_{1, k}\right)^{2}+\left(y_{1 s, k}+n_{2, k}\right)^{2}\right. \\
& \left.+q_{1, k}^{2}+q_{2, k}^{2}\right] \\
v^{-}= & \frac{1}{2} \sum_{k=0}^{\infty} \lambda_{k}\left[\left(y_{2 c, k}+n_{3, k}\right)^{2}+\left(y_{2 s, k}+n_{4, k}\right)^{2}\right. \\
& \left.+q_{3, k}^{2}+q_{4, k}^{2}\right]
\end{aligned}
$$

where the noise terms $q_{l, k}$, for $l \in\{1, \ldots, 4\}$, are statistically distributed as the terms $n_{l, k}$, for $l \in\{1, \ldots, 4\}$, and are independent of them.

\section{Evaluation of the ERror Probability}

\section{A. Moment Generating Function Derivation}

The statistics of the random variable $v$ are described here using the MGF, which is defined as $M_{v}(s)=\left\langle e^{s v}\right\rangle$. This function is computed by deriving, in first place, the conditional MGF of $v$ for a given realization of $\Delta \theta=\left[\Delta \theta_{1}, \Delta \theta_{2}, \ldots, \Delta \theta_{N}\right]$, denoted as $M_{v \mid \Delta \theta}(s)$, and then averaging over all the possible values of $\Delta \theta$. From (4), (13), and (14), it can be concluded that the conditional random variable $v \mid \Delta \theta$ is the difference of two non-central chi-square variables. In these circumstances, as the cross-correlation coefficient $r_{j, l}=\left\langle n_{j, k} n_{l, k}\right\rangle$ between the noise terms $n_{j, k}$ and $n_{l, k}$ is equal to $r_{j, l}=\delta_{j l}$, the non-central chi-squares are independently distributed [37], [38], and in this way the conditional MGF of $v, M_{v \mid \Delta \theta}(s)$, can be written as the product of the MGFs of the conditional random variables $v^{+} \mid \Delta \theta$ and $v^{-} \mid \Delta \theta$. As a consequence, the unconditional MGF of $v$ is given by

$$
M_{v}(s)=M_{v^{+}}(s) M_{v^{-}}(-s)
$$


where

$M_{v^{+}}(s)=\frac{1}{\prod_{k=0}^{\infty}\left[1-s \lambda_{k} N_{0} / 2\right]^{\rho}} M_{y_{1}}\left[\sum_{k=0}^{\infty} \frac{s \lambda_{k} T \xi_{k}}{\left(1-s \lambda_{k} N_{0} / 2\right)}\right]$

$M_{v^{-}}(s)=\frac{1}{\prod_{k=0}^{\infty}\left[1-s \lambda_{k} N_{0} / 2\right]^{\rho}} M_{y_{2}}\left[\sum_{k=0}^{\infty} \frac{s \lambda_{k} T \xi_{k}}{\left(1-s \lambda_{k} N_{0} / 2\right)}\right]$

with

$$
\begin{aligned}
M_{y_{1}}(s)= & \exp \left(s G P_{s} \alpha_{s}^{+}\right) \\
& \times \prod_{i=1}^{N} \exp \left(s G P_{s} \varepsilon_{i} \alpha_{x, i}^{+}\right) I_{0}\left(s 2 G P_{s} \sqrt{\varepsilon_{i} \alpha_{s}^{+} \alpha_{x, i}^{+}}\right) \\
& \times \prod_{j=1}^{N-1} \prod_{i=j+1}^{N} I_{0}\left(s 2 G P_{s} \sqrt{\varepsilon_{j} \varepsilon_{i} \alpha_{x, j}^{+} \alpha_{x, i}^{+}}\right)
\end{aligned}
$$

with $I_{0}(\cdot)$ denoting the modified Bessel function of the first kind of order zero and $\xi_{k}=u_{k}^{2} / T$, whereas $M_{y_{2}}(s)$ is given by (27) with $\alpha_{s}^{+}$replaced by $\alpha_{s}^{-}$and $\alpha_{x, i(j)}^{+}$replaced by $\alpha_{x, i(j)}^{-}$, and $\rho=1$. When the ASE noise in the orthogonal polarization is accounted for (25) and (26) still hold, but now with $\rho=2$. The way how (25) to (26) were derived deserves some attention. First of all $v^{+}$and $v^{-}$are a sum of noncentral chi-square random variables, whose individual MGFs are well known [39]. As these random variables are statistically independent, then the final MGFs of $v^{+}$and $v^{-}$are the product of the individual ones. The next step of the derivation process requires the averaging over $\Delta \theta$, which leads to $M_{y_{1}}(s)$. This function is obtained from $M_{y_{1 k}}(s)$, deduced in Appendix, by changing the variable $s$ by $s^{\prime} u_{k}^{2}$. In this derivation, for the sake of simplicity, we have assumed that the crosstalk-crosstalk beating terms are independent of the signal-crosstalk beating terms.

A concurring technique for MGF representation used by some authors in crosstalk studies on OOK scenarios [40], [41] relies on the wideband optical filtering assumption. This approximation avoids the problem of solving complicated integral equations by using, for example, complex exponentials as orthogonal functions in the signal and noise expansions. The technique has also been adapted to DPSK [25], [26], and implies using an ideal optical filter with a large bandwidth-time product $\left(B_{O} T\right)$ and an integrate-and-dump electrical filter with $h_{e}(t)=1 / T$ for $t \in[0, T]$ and zero elsewhere. In this case, the MGFs given by (25) and (26) can be simplified by making the following approximations: 1) $\lambda_{k}=1 / T$, for $k<B_{o} T$ and zero elsewhere; 2) $\sum_{k=0}^{B_{o} T-1} \xi_{k}=1$. Intuitively, it is expected that the accuracy of the referred simplification will depend on both the filters profile and bandwidth. To analyze this problem the eigenfunctions $\left\{\varphi_{k}(t)\right\}$ and the eigenvalues $\left\{\lambda_{k}\right\}$ of (11) are obtained for two different receiver configurations: 1) Ideal receiver, resulting from the combination of an ideal optical filter and an electrical integrate-and-dump filter; 2) Gaussian receiver, based on Gaussian shaped optical and electrical filters. The integral equation (11) was solved by applying the Gaussian quadrature rules integration technique and the resultant matrix eigenvalue problem was solved by applying the QL algorithm with implicit shifts [42], which requires that the matrix kernel is

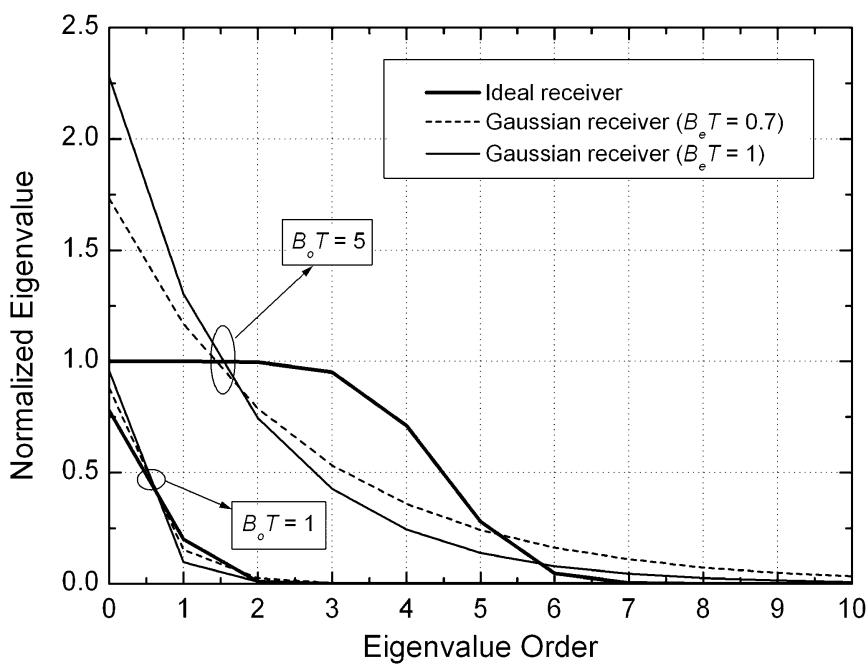

Fig. 2. Normalized eigenvalues $\lambda_{k}^{\prime}$ corresponding to the ideal and Gaussian receiver cases.

first transformed into a symmetric and tridiagonal matrix [42]. Fig. 2 represents the normalized eigenvalues $\lambda_{k}^{\prime}=T \lambda_{k}$ for the receiver configurations considered. As shown, for $B_{o} T=1$ all the eigenvalues $\lambda_{k}^{\prime}$ are smaller than 1 , indicating that the first requirement of the simplified approach does not hold. As an additional remark, it should be referred that this discrepancy is higher for the ideal receiver $\left(\lambda_{0}=0.783\right)$ than for the Gaussian receiver $\left(\lambda_{0}=0.879\right.$ for $B_{e} T=0.7$ and $\lambda_{0}=0.957$ for $\left.B_{e} T=1\right)$. For $B_{o} T=5$ the results corresponding to the ideal receiver are in accordance with the first requirement of the wideband assumption, whereas the results for the Gaussian receiver clearly depart from that requirement.

Using the eigenfunctions $\left\{\varphi_{k}(t)\right\}$ and applying (21) the parameter $\xi_{k}$ can be computed. The knowledge of $\xi_{k}$ permits to evaluate $\sum_{k} \xi_{k}$, which value is represented in Fig. 3 as a function of $B_{O} T$. The summation value approximates 1 as $B_{o} T$ increases, but deviates markedly from this value for low values of $B_{o} T$, indicating that the simplified approach lacks accuracy in this region. This trend is more remarkable for the ideal receiver where $B_{o} T=1$ leads to $\sum_{k} \xi_{k}=0.782$, whereas for the Gaussian receiver we have $\sum_{k} \xi_{k}=0.9$ for both $B_{e} T=0.7$ and $B_{e} T=1$. These results suggest that for what concerns the statistical modeling of the decision variable the wideband approximation lacks accuracy when the Gaussian receiver is used, restricting its range of application only to scenarios based on the ideal receiver with bandwidth-time products larger than 1 .

\section{B. Exact Error Probability Evaluation}

From (24) the probability density function (PDF) of the decision variable can be evaluated by using the inverse Laplace transform, that is [43]

$$
p_{v}(v)=\frac{1}{2 \pi j} \int_{c-j \infty}^{c+j \infty} M_{v}(-s) e^{s v} d s
$$

in which $s=x+j \omega$, and the contour of integration is a vertical line parallel to the imaginary $s$ axis that crosses the real $s$ axis at the point $c$, that lies in the region in which the integral converge.

The correct detection of binary information digits depends on the observed sign of the random variable $v$. When signaling 


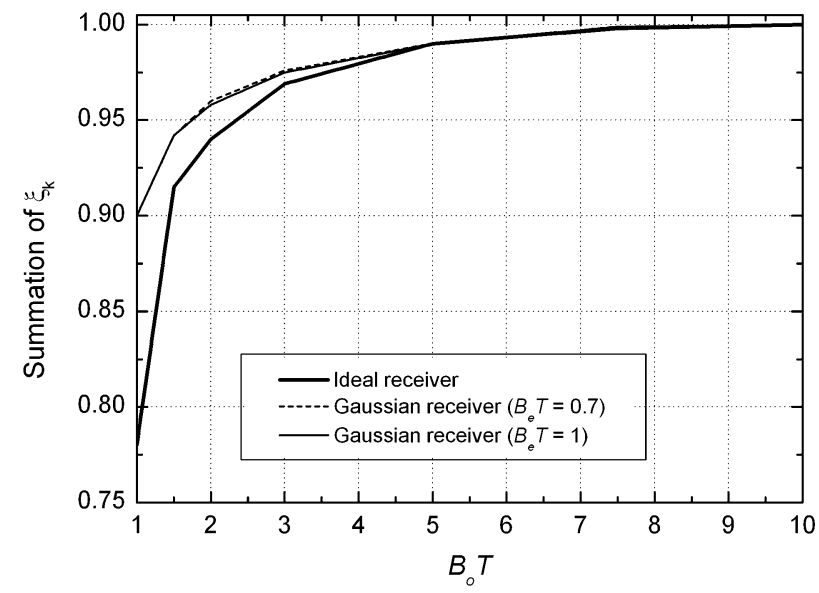

Fig. 3. Summation of all $\xi_{k}$ as a function of the bandwidth-time product $B_{o} T$ for the ideal and Gaussian receiver.

a "one" in a DPSK signal the observed sign of $v$ should be positive. Taking into consideration the fact that the optimum decision threshold for an ideal balanced receiver is zero [24], then in that situation an observed negative value leads to a decision error. As a consequence, the error probability can be evaluated by integrating (28) from $-\infty$ to 0 and can be written, for the specific case that $k$ of the $N$ interferers are "ones", in the following form:

$$
\begin{aligned}
P_{e}(k) & =P_{r}\left(v<0 \mid a_{s}=1, k\right)=\int_{-\infty}^{0} p_{v}\left(v \mid a_{s}=1, k\right) d v \\
& =\frac{1}{2 \pi j} \int_{c-j \infty}^{c+j \infty} \frac{M_{v}\left(-s \mid a_{s}=1, k\right)}{s} d s, 0<c<c_{1}
\end{aligned}
$$

where $P_{r}(\cdot)$ denotes probability, $c_{1}$ is the upper limit of the region of convergence of the integral, and $M_{v}\left(s \mid a_{s}=1, k\right)$ is the conditional MGF given that the signal symbol is "one" and the number of interferers at the state "one" is $k$. Bearing in mind that for $k$ interferers in the state "one" there are $N-k$ in the state "zero", and using (24), the conditional MGF is $M_{v}(s \mid k)=$ $M_{v^{+}}(s \mid k) M_{v^{-}}(-s \mid N-k)$. The average error probability is evaluated by applying a binomial symbol conditioning on the interfering signals. As a result, and for the case of the "one" and "zero" interfering symbols being equally likely, the average error probability is given by

$$
P_{e}=\frac{1}{2^{N+1}} \sum_{k=0}^{N}\left(\begin{array}{c}
N \\
k
\end{array}\right) P_{e}(k) .
$$

The integral in (29) can be evaluated numerically by using the saddle point integration method [44]. According to this method a possible contour of integration is a straight line parallel to the imaginary $s$ axis, which is required to pass through the saddle point of the integrand of (29) on the positive real $s$ axis. This saddle point, designated as $s_{0}$, is the solution of the equation $\psi^{\prime}(s)=0$, this is the root of the first-order derivative of the phase function

$$
\psi(s)=\ln M_{v}\left(-s \mid a_{s}=1, k\right)-\ln s .
$$

It should be noted that the same strategy can be used to solve the integral in (28) in order to obtain the PDF of the decision variable $v$, although in this case the phase function (31) must be replaced by $\psi(s)=\ln M_{v}(-s)+s v$.

Expanding $\psi(s)$ into a Taylor series around the point $s_{0}$, and retaining only the terms up to order $s^{2}$, one arrives to the wellknown saddle-point approximation. This approximation gives quite accurate results in the context of error probability estimations, but fails to describe properly the probability densities of DPSK signals in the presence of crosstalk, especially in the center region of the curves [45]. For this reason, and for a question of homogeneity in numerical calculations, we have only used the saddle point integration method through all this paper in order to compute both probability densities and error probabilities.

\section{ILLUSTRATING RESULTS}

In order to illustrate the application of the rigorous formulation developed previously, this section presents some numerical results for both the ideal and the Gaussian receiver configurations, assuming a bit rate of $10 \mathrm{~Gb} / \mathrm{s}$ and a Gaussian electrical filter with a $3 \mathrm{~dB}$ bandwidth such that $B_{e} T=0.7$. Furthermore, the analysis takes into account the impact of in-band crosstalk and ASE noise from both polarizations. This noise is due to the optical pre-amplifier, which is characterized by $G=30 \mathrm{~dB}$ and $F=5 \mathrm{~dB}$. For comparison purposes, the simplified approach resulting from the optical filtering wideband assumption is also evaluated.

We start our discussion with the evaluation of the PDF of the decision variable $p_{v}(v)$, since the precise knowledge of this function is crucial to gain insight into DPSK properties. In these evaluations, it is assumed that the desired signal and the interferers are in the same symbol state $\left(a_{s}=a_{x, i}\right.$, with $i \in$ $\{1, \ldots, N\})$, and that the average signal power incident at optical pre-amplifier input is $-40 \mathrm{dBm}$. Fig. 4 shows the PDFs of the decision variable (normalized with respect to the signal power $P_{s}$ ), for the two receiver configurations, considering a single interferer with a crosstalk level of $-15 \mathrm{~dB}$. The results for the simplified approach are also included. It is clearly seen from Fig. 4 that for the different scenarios considered the inner tails of the PDFs associated with the symbols "one" and "zero" are symmetric around zero. This is a distinctive feature of DPSK, provided that the optical demodulator is ideal [32], and explains why its decision threshold is zero. Fig. 4(a), which corresponds to the case of $B_{o} T=1$, also shows that the crossing point of the ideal receiver curves occurs at a higher value than the other curves, but this situation changes for $B_{o} T=5$ (Fig. 4(b)) with the simplified approach and ideal receiver curves being almost indistinguishable and the crossing point of the curves corresponding to the Gaussian receiver leading to the highest value. As the error probability is determined by the area under the inner tails of the PDFs, it is expected that the simplified formulation resulting from the wideband assumption leads only to accurate performance estimations for the ideal receiver with a bandwidth-time product larger than 1, confirming the conclusions derived from the analysis presented in Section III.A. Fig. 5 shows again the PDFs of the decision variable, but only for the Gaussian receiver, considering a total crosstalk level of -15 $\mathrm{dB}$ equally distributed among a variable number of interferers. 


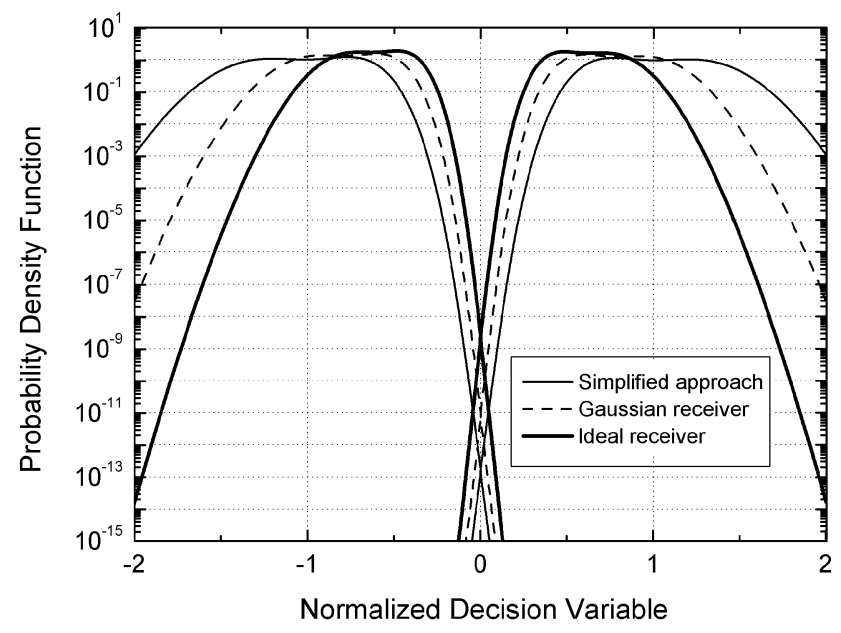

(a)

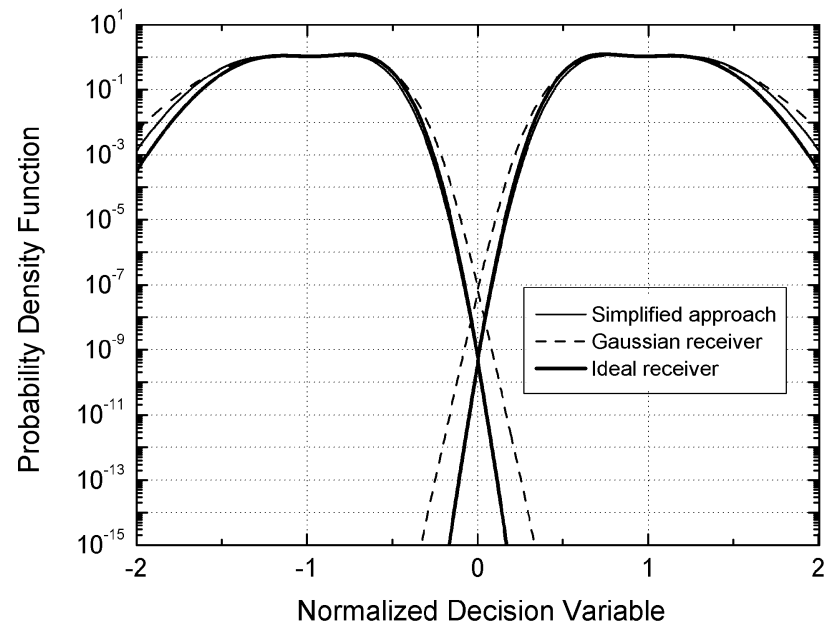

(b)

Fig. 4. Probability density function of the normalized decision variable for both "one" and "zero" symbols, for two receiver configurations, considering a single interferer and the following parameters: $P_{s}=-40 \mathrm{dBm}, \varepsilon=-15 \mathrm{~dB}$. (a) $B_{o} T=1$; (b) $B_{o} T=5$.

As expected, the crossing points increase with the number of interferers and their values are higher for the $B_{o} T=5$ case (Fig. 5(b)) than for the $B_{o} T=1$ one (Fig. 5(a)). This behavior is due to the fact that the impact of the ASE noise is more significant for $B_{o} T=5$ than for $B_{o} T=1$, leading to an enlargement of the PDF shapes, as can be easily confirmed by comparing Fig. 5(a) with Fig. 5(b).

The effect of the number of interferers on the receiver performance is shown in Figs. 6(a) and (b) for $B_{o} T=1$ and $B_{o} T=$ 5 , respectively, considering a total crosstalk level of $-15 \mathrm{~dB}$. These figures plot the error probability as a function of the optical signal-to-noise ratio (OSNR) for the two receiver configurations under consideration, which is computed using the exact analysis described in the previous sections. The error probability evaluated using the simplified approximation resulting from the wideband assumption is also plotted. The OSNR is defined in this work as the ratio of the average signal power before the optical filter to the ASE noise power in both polarizations evaluated using a noise bandwidth equal to the bit rate. As the first observation, it may be noted that the curve for the single interferer in Fig. 6(a), computed with the simplified approximation,

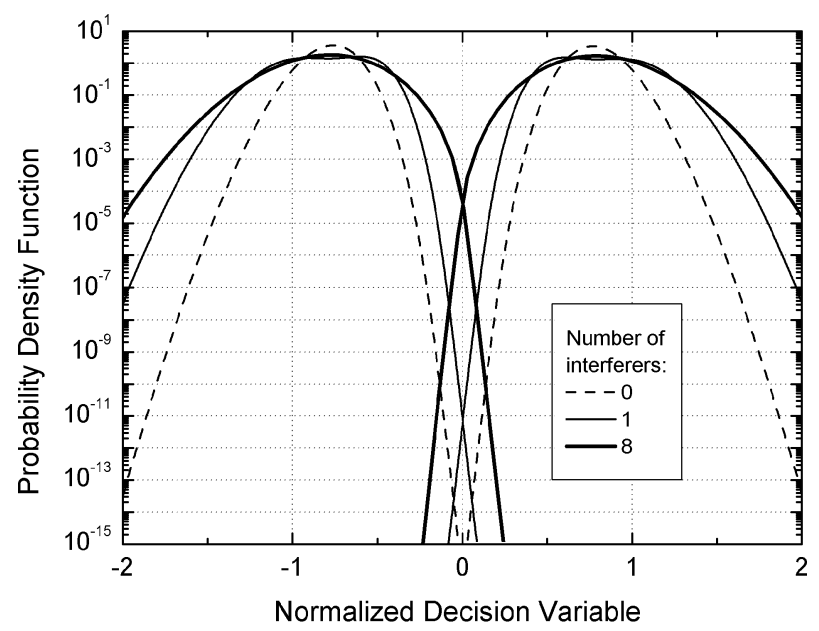

(a)

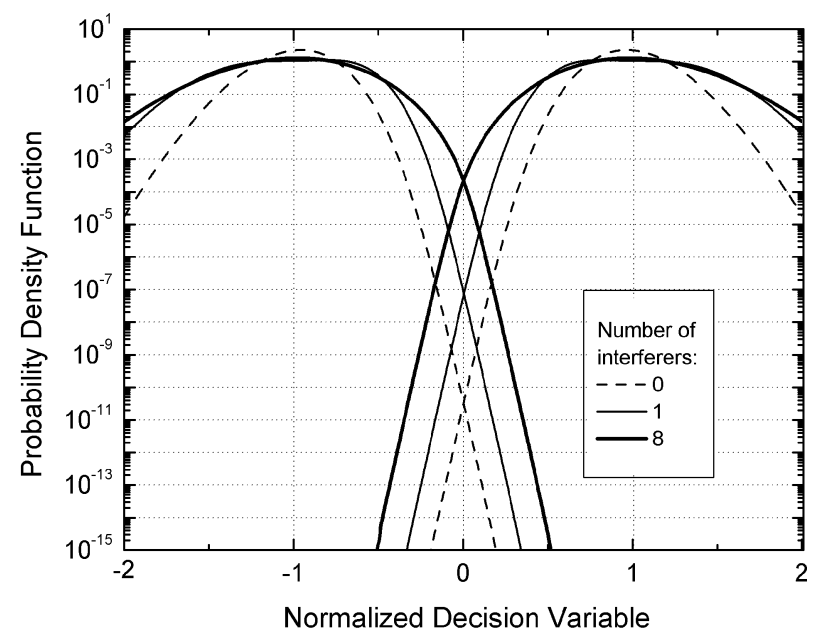

(b)

Fig. 5. Probability density function of the normalized decision variable for both "one" and "zero" symbols, for the Gaussian receiver with $B_{e} T=0.7$, considering different number of interferers and the parameters: $P_{s}=-40 \mathrm{dBm}$, and $\varepsilon_{T}=-15 \mathrm{~dB}$. (a) $B_{o} T=1$; (b) $B_{o} T=5$.

is in excellent agreement with that obtained using ([23], (20)) or ([46], (8.30)). Several other observations can be made. In first place, it is clear that despite the total crosstalk level remaining constant $\left(\varepsilon_{T}=-15 \mathrm{~dB}\right)$ the performance deteriorates as the number of interferers increases, a trend that is already expected from the analysis of the PDF behavior. The curves also show that the performance depends on the shape of the optical and electrical filters, with the Gaussian receiver with $B_{o} T=5$ experiencing about $1.0 \mathrm{~dB}$ and $0.7 \mathrm{~dB}$ penalties with respect to the ideal one at $P_{e}=10^{-9}$ for $N=1$ and $N=8$, respectively. On the other hand, for the $B_{o} T=1$ case, the discrepancies between the results obtained with the two receiver configurations are reduced and the referred penalties decrease to 0.5 $\mathrm{dB}$. In both figures the presence of an error floor is clearly evident for both $N=8$ and $N=16$. This floor is due to the in-band crosstalk, as has already been evidenced in [24], and in the case of $N=16$ it even prevents to reach an error probability of $10^{-9}$. Fig. 6(a) also indicates that for $B_{o} T=1$ the simplified approach is not appropriate to provide accurate performance estimates for neither the ideal receiver, nor the Gaussian receiver, leading to an OSNR penalty of about $1.1 \mathrm{~dB}$ at $P_{e}=10^{-9}$ for 


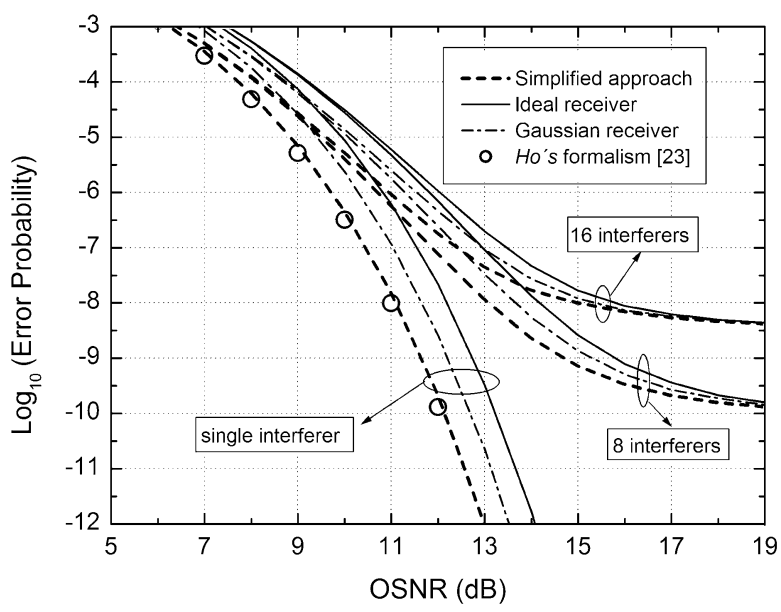

(a)

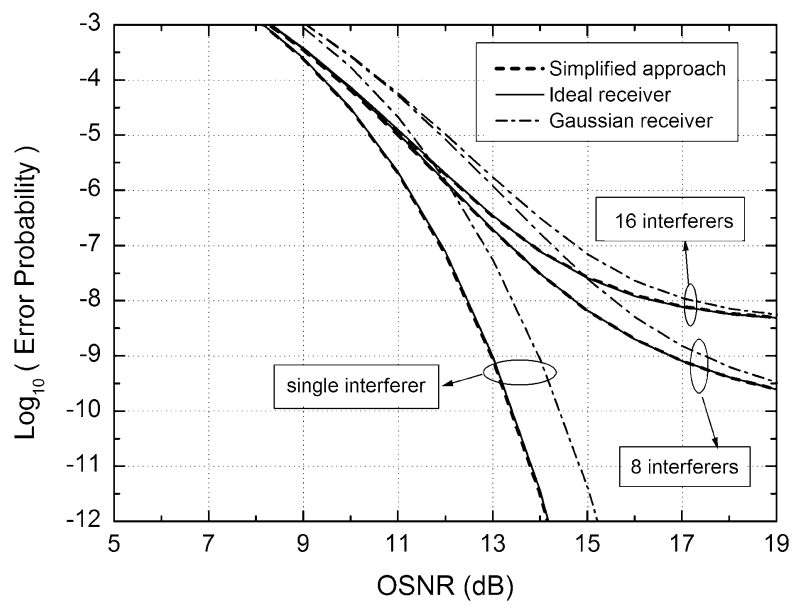

(b)

Fig. 6. Error probability as a function of the OSNR for a total crosstalk level of $-15 \mathrm{~dB}$ and two receiver configurations with the number of interferers as a parameter. (a) $B_{o} T=1$; (b) $B_{o} T=5$.

the former receiver and $0.6 \mathrm{~dB}$ for the latter one, assuming again that $N<16$. For $B_{o} T=5$ the results obtained with the simplified analysis are in good agreement with the ones obtained using the exact treatment for the ideal receiver, but the approximation also fails to provide rigorous estimates for the Gaussian receiver. Indeed, in this case the discrepancy between the exact and simplified results is about $1.0 \mathrm{~dB}$ and $0.7 \mathrm{~dB}$ for $N=1$ and $N=8$, respectively, assuming the same conditions as before. Therefore, the simplified approach is not a reliable method for predicting error probabilities for Gaussian receivers in the presence of ASE noise and in-band crosstalk, and also fails for ideal receivers with bandwidth-time products closer to 1 .

Next, we consider in Fig. 7 the results for the OSNR penalty due to in-band crosstalk as a function of the total crosstalk level $\varepsilon_{T}$, considering $B_{o} T=5$. The OSNR penalty is a widely used metric to quantify the crosstalk impact and is defined in this work as the increment in decibels in the OSNR, required to maintain the error probability fixed at $10^{-9}$ in the presence of crosstalk. Contrary to what happened in the error probability analysis, the OSNR penalty results are practically independent of the filter shapes. These results, as well as our calculations for other values of $B_{o} T$, also evidence the fact that the simplified

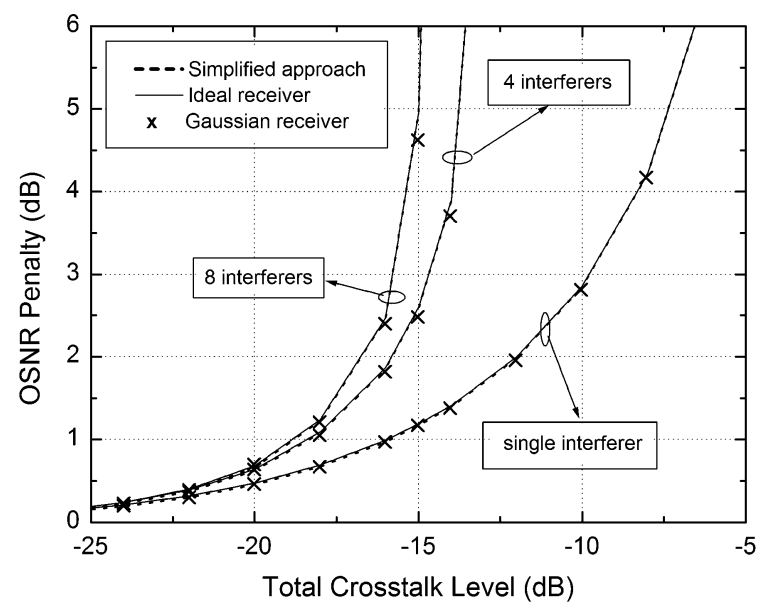

Fig. 7. OSNR penalty versus the total crosstalk level for different numbers of interferers and two receiver configurations. The error probability is fixed at $10^{-9}$ and $B_{o} T=5$.

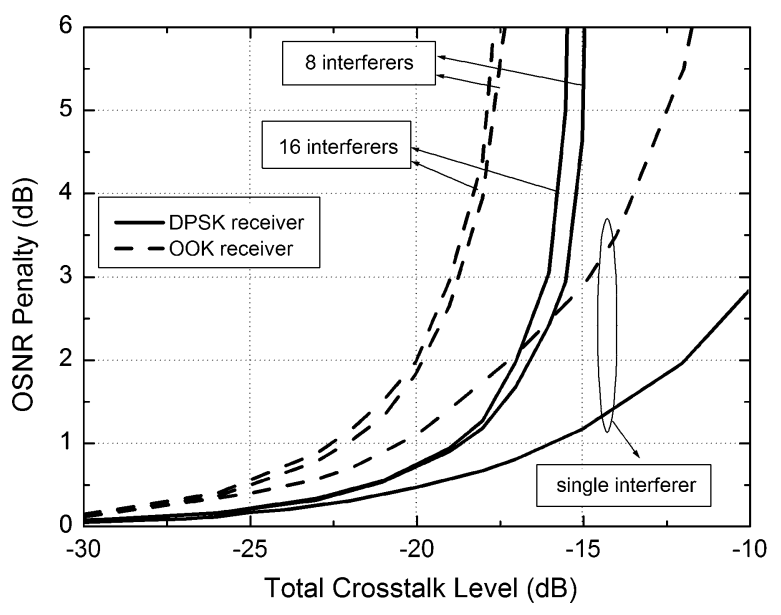

Fig. 8. OSNR penalty as a function of the total crosstalk level for both DPSK and $\mathrm{OOK}$ with the number of interferers as a parameter, considering a Gaussian receiver with $B_{o} T=5$. The error probability is fixed at $10^{-9}$.

approach can be employed to obtain accurate penalties irrespectively of the bandwidth-time product.

Finally, in order to compare the penalties of the DPSK format with the ones of the OOK modulation with NRZ pulses, we estimated the performance of OOK, following the same lines outlined in the previous sections adapted to an OOK receiver. A particular difference between the models resides on accounting for OOK the effect of the extinction ratio, which is defined as the ratio of the average optical power of symbol "one" and that of the symbol "zero", assumed in this paper to be equal to $20 \mathrm{~dB}$. The obtained results are plotted in Fig. 8, showing that for a 2 $\mathrm{dB}$ OSNR penalty and a single interferer case DPSK has a gain of $5.5 \mathrm{~dB}$ over OOK in terms of crosstalk tolerance, since it tolerates a $-11.9 \mathrm{~dB}$ crosstalk level, whereas OOK only tolerates $\mathrm{a}-17.4 \mathrm{~dB}$. Note that this gain is very close to the $6 \mathrm{~dB}$ one, reported by Liu et al. [17], obtained by experimental means. For the case of 8 and 16 interferers, the crosstalk tolerance is reduced to about $3.2 \mathrm{~dB}$ and $3.0 \mathrm{~dB}$, respectively, demonstrating that the robustness of DPSK towards in-band crosstalk diminishes as the number of interferers increases. 


\section{CONCLUSION}

A rigorous formulation has been developed in this paper to study optically pre-amplified DPSK receivers in the presence of in-band crosstalk due to an arbitrary number of interferers. The formulation relies on using an eigenfunction expansion technique and is sufficiently general to deal with the case of arbitrary optical and electrical filtering. The method was used afterwards to analyze the accuracy of the wideband optical filtering assumption. Numerical results show that the error probability estimates depend on the filter shapes, and also suggest that the wideband assumption lacks accuracy when realistic receiver configurations, like the one resulting from the combination of Gaussian optical and electrical filters, are used, restricting its range of application only to scenarios based on an ideal receiver with bandwidth-time products larger than 1 .

However, the situation is quite different for the OSNR penalty due to in-band crosstalk, the metric commonly use to quantify the crosstalk effects. In this case, the results remain almost independent of the filters profile and the wideband approximation can be used to give reliable predictions to this metric. In addition, the crosstalk tolerance of DPSK over OOK was also analyzed and shown that the advantages of using DPSK reduce as the number of interferers increases.

\section{APPENDIX}

In this Appendix, the MGF of the random variable $y_{1, k}^{2}$, which will permit to obtain (27), is derived. This function can be written as

$$
\begin{aligned}
M_{y_{1, k}}\left(s^{\prime}\right) & =\left\langle\exp \left(s^{\prime} y_{1, k}^{2}\right)\right\rangle \\
& =\left\langle\exp \left[s^{\prime}\left(y_{1 c, k}^{2}+y_{1 s, k}^{2}\right)\right] / 2\right\rangle
\end{aligned}
$$

Taking into account (19) and (20) we can write

$$
\begin{aligned}
& \frac{1}{2}\left(y_{1 c, k}^{2}+y_{1 s, k}^{2}\right) \\
& =G P_{s} \alpha_{s}^{+} u_{k}^{2}+\mathrm{G} \sum_{i=1}^{N} P_{x, i} \alpha_{x, i}^{+} u_{k}^{2} \\
& \quad+2 G \sum_{i=1}^{N} \sqrt{P_{s} P_{x, i} \alpha_{s}^{+} \alpha_{x, i}^{+}} u_{k}^{2} \cos \left(\Delta \theta_{i}\right) \\
& \quad+2 G \sum_{j=1}^{N-1} \sum_{i=j+1}^{N} \sqrt{P_{x, i} P_{x, j} \alpha_{x, i}^{+} \alpha_{x, j}^{+}} u_{k}^{2} \\
& \quad \times \cos \left(\Delta \theta_{i}-\Delta \theta_{j}\right) .
\end{aligned}
$$

Inspecting the right-hand side part of (A2) we verify that the first and the second terms are the optical power of the signal and crosstalk, respectively. The third term corresponds to the signal-crosstalk beating, and the last one to the crosstalk-crosstalk beating. The presence of the random variable $\Delta \theta_{i}$ in both the beating terms introduces some correlation between the signal-crosstalk and crosstalk-crosstalk terms, making the expectation in (A1) particularly difficult to evaluate in an exact way. To simplify the calculations we assumed that the terms of the crosstalk-crosstalk contribution are mutually independent and uncorrelated from the terms of the signal-crosstalk contribution. In this case, if we have in mind that $\Delta \theta_{i}$ is uniformly distributed in $[0,2 \pi]$ and $P_{x, i}=\varepsilon_{i} P_{s}$, then (A1) can be written as

$$
\begin{aligned}
M_{y_{1, k}}\left(s^{\prime}\right) & \\
= & \exp \left(s^{\prime} G P_{s} \alpha_{s}^{+} u_{k}^{2}\right) \prod_{i=1}^{N} \exp \left(s^{\prime} G P_{s} u_{k}^{2} \varepsilon_{i} \alpha_{x, i}^{+}\right) \\
& \times \int_{0}^{2 \pi} \exp \left[s^{\prime} 2 G P_{s} u_{k}^{2} \sqrt{\varepsilon_{i} \alpha_{s}^{+} \alpha_{x, i}^{+}} \cos \left(\Delta \theta_{i}\right)\right] d \Delta \theta_{i} \\
& \times \prod_{j=1}^{N-1} \prod_{i=j+1}^{N} \int_{0}^{2 \pi} \exp \left[s^{\prime} 2 G P_{s} u_{k}^{2} \sqrt{\varepsilon_{i} \varepsilon_{j} \alpha_{x, j}^{+} \alpha_{x, i}^{+}}\right. \\
& \left.\times \cos \left(\Delta \theta_{i}-\Delta \theta_{j}\right)\right] d\left(\Delta \theta_{i}-\Delta \theta_{j}\right) .
\end{aligned}
$$

Having in mind that the expected value of $\exp \left(s^{\prime} \cos \left(\Delta \theta_{i}\right)\right)$ is $I_{0}\left(s^{\prime}\right)([47],(3.339))$ the above expression can be written as

$$
\begin{aligned}
M_{y_{1}, k}\left(s^{\prime}\right)= & \exp \left(s^{\prime} G P_{s} \alpha_{s}^{+} u_{k}^{2}\right) \\
& \times \prod_{i=1}^{N} \exp \left(s^{\prime} G P_{s} u_{k}^{2} \varepsilon_{i} \alpha_{x, i}^{+}\right) \\
& \times I_{0}\left(s^{\prime} 2 G P_{s} u_{k}^{2} \sqrt{\varepsilon_{i} \alpha_{s}^{+} \alpha_{x, i}^{+}}\right) \\
& \times \prod_{j=1}^{N-1} \prod_{i=j+1}^{N} I_{0}\left(s^{\prime} 2 G P_{s} u_{k}^{2} \sqrt{\varepsilon_{j} \varepsilon_{i} \alpha_{x, j}^{+} \alpha_{x, i}^{+}}\right) .
\end{aligned}
$$

It is worth noting that the impact of the crosstalk-crosstalk components grows as the OSNR increases. This effect is more visible in the scenarios depicted in Figs. 6(a) and (b), especially for the case of $N=8$. If we neglect those components we find out that there is no noticeable error floor for the case referred. However, in the presence of the crosstalk-crosstalk beating terms an error floor is building up, a phenomenon that is especially evident in Fig. 6(a). Besides this aspect, the influence of these terms is minimal in all the other results presented in Section IV.

\section{REFERENCES}

[1] K. Emura, M. Shikada, S. Yamazaki, K. Komatsu, I. Mito, and K. Minemura, "Optical DPSK heterodyne detection experiments using DBR laser diodes with external optical feedback," Electron. Lett., vol. 21, no. 24, pp. 1121-1122, Nov. 1985.

[2] G. Jacobsen and I. Garret, "Theory for optical heterodyne DPSK receivers with post-detection filtering," J. Lightw. Technol., vol. LT-5, no. 4, pp. 478-484, Apr. 1987.

[3] R. S. Vodhanel, A. F. Elrefaie, M. Z. Iqbal, R. E. Wagner, J. L. Gimlett, and S. Tsuji, "Performance of directly modulated DFB lasers in 10-Gb/s ASK, FSK, and DPSK," J. Lightw. Technol., vol. 8, no. 9, pp. 1379-1386, Sep. 1990.

[4] J. J. O. Pires and J. R. F. da Rocha, "Performance analysis of DPSK direct detection optical systems in the presence of interferometric intensity noise," J. Lightw. Technol., vol. 10, no. 11, pp. 1722-1730, Nov. 1992.

[5] T. J. Paul, E. A. Swanson, J. C. Livas, R. S. Bondurant, and R. J. Magliocco, "3 Gbit/s optically preamplified direct detection DPSK receiver with 116 photon/bit sensitivity," Electron. Lett., vol. 29, no. 7, pp. 614-615, Apr. 1993.

[6] A. H. Gnauck et al., " $2.5 \mathrm{~Tb} / \mathrm{s}(64 \times 42.7 \mathrm{~Gb} / \mathrm{s})$ transmission over $40 \times 100 \mathrm{~km}$ NZDSF using RZ-DPSK format and all-Raman-amplified spans," in Proc. OFC2002, Anaheim, CA, Mar. 2002, Post-deadline paper FC2. 
[7] J.-X. Cai et al., "A DWDM demonstration of $3.73 \mathrm{~Tb} / \mathrm{s}$ over $11000 \mathrm{~km}$ using 373 RZ-DPSK channels at $10 \mathrm{~Gb} / \mathrm{s}$," in Proc. OFC 2003, Atlanta, GA, Mar. 2003, Post-deadline paper PD22.

[8] D. G. Foursa, "DPSK performance in field and laboratory experiments," in Proc. OFC/NFOEC, 2005, Anaheim, CA, Mar. 2005, paper OThC3.

[9] J. Wang and J. M. Kahn, "Impact of chromatic and polarization-mode dispersions on DPSK systems using interferometric demodulation and direct detection," J. Lightw. Technol., vol. 22, no. 2, pp. 362-371, Feb. 2004.

[10] A. H. Gnauck and P. J. Winzer, "Optical phase-shift-keyed transmission," J. Lightw. Technol., vol. 23, no. 1, pp. 115-130, Jan. 2005.

[11] J. J. O. Pires and M. O'Mahony, "Photonic switch size limitations imposed by phase noise from semiconductor optical amplifier gates," Electron. Lett., vol. 31, no. 18, pp. 1582-1584, Aug. 1995.

[12] O. Liboiron-Ladoucer, R. Jordan, and K. Bergman, "10 Gbps NRZDPSK modulation in SOA-based optical packet switching networks," in Proc. ECOC2005, Glasgow, U.K., Sep. 2005, paper Th2.4.3.

[13] N. Chi et al., "Experimental characteristics of optical crosspoint switch matrix and its applications in optical packet switching," $J$. Lightw. Technol., vol. 24, no. 10, pp. 3646-3653, Oct. 2006.

[14] H. Nishizawa, Y. Yamada, Y. Shibata, and K. Habara, "10-Gb/s optical DPSK packet receiver proof against large power fluctuations," IEEE Photon. Technol. Lett., vol. 11, no. 6, pp. 733-735, Jun. 1999.

[15] Y. Su, X. Liu, and J. Leuthold, "Wide dynamic range 10-Gb/s DPSK packet receiver using optical-limiting amplifiers," IEEE Photon. Technol. Lett., vol. 16, no. 1, pp. 296-298, Jan. 2004.

[16] P. J. Winzer, S. Chandrasekhar, and H. Kim, "Impact of filtering on RZ-DPSK reception," IEEE Photon. Technol. Lett., vol. 15, no. 6, pp. 840-842, Jun. 2003.

[17] X. Liu, Y.-H. Kao, M. Movassaghi, and R. C. Giles, "Tolerance to in-band coherent crosstalk of differential phase-shift-keyed signal with balanced detection and FEC," IEEE Photon. Technol. Lett., vol. 16, no. 4, pp. 1209-1211, Apr. 2004.

[18] S. D. Dods and R. S. Tucker, "A comparison of the homodyne crosstalk characteristics of optical add-drop multiplexers," J. Lightw. Technol., vol. 19, no. 12, pp. 1829-1838, Dec. 2001.

[19] T. Y. Chai, T. H. Cheng, Y. Ye, and Q. Liu, "Inband crosstalk analysis of optical cross-connect architectures," J. Lightw. Technol., vol. 23, no. 2, pp. 688-701, Feb. 2006.

[20] H. Buchta, E. Patzak, and J. Saniter, "Limits of effective throughput of optical burst switches based on semiconductor optical amplifiers," in Proc. OFC2003, Atlanta, Georgia, Mar. 2003, pp. 215-217.

[21] E. L. Goldstein and L. Eskildsen, "Scaling limitations in transparent optical networks due to low-level crosstalk," IEEE Photon. Technol. Lett., vol. 7, no. 1, pp. 93-94, Jan. 1995.

[22] J. C. Attard, J. E. Mitchell, and C. J. Rasmussen, "Performance analysis of interferometric noise due to unequally powered interferers in optical networks," J. Lightw. Technol., vol. 23, no. 4, pp. 1692-1703, Apr. 2005.

[23] K.-P. Ho, "Analysis of direct-detection DPSK signal with homodyne crosstalk," J. Lightw. Technol., vol. 23, no. 9, pp. 2681-2686, Sep. 2005.

[24] T. Kamalakis and T. Sphicopoulos, "Performance analysis of differential-phase-shift-keying optical receivers in the presence of in-band crosstalk noise," J. Lightw. Technol., vol. 23, no. 12, pp. 4084-4092, Dec. 2005

[25] L. G. C. Cancela and J. J. O. Pires, "Impact of intrachannel crosstalk on the performance of direct-detection DPSK optical systems," in Proc. CLEO2005, Baltimore, MD, May 2005, paper JthE48.

[26] L. G. C. Cancela and J. J. O. Pires, "Numerical study on the tolerance to intrachannel crosstalk of direct-detection DPSK optical systems," in Proc. ECOC 2005, Glasgow, Scotland, Sep. 2005, pp. 711-712.

[27] D. Marcuse, "Derivation of analytical expressions for the bit-error probability in lightwave systems with optical amplifiers," J. Lightw. Technol., vol. 8, no. 12, pp. 1816-1823, Dec. 1990.

[28] P. A. Humblet and M. Azizoglu, "On the bit error rate of lightwave systems with optical amplifiers," J. Lightw. Technol., vol. 9, no. 11, pp. 1576-1582, Nov. 1991.

[29] L. G. C. Cancela and J. J. O. Pires, "On the accuracy of the Gaussian approximation for performance estimation in optical DPSK systems with in-band crosstalk," in Proc. IEEE ICC 2007, Glasgow, U.K., Jun. 2007, pp. 2474-2479.
[30] J. J. O. Pires and L. G. C. Cancela, "A contribution to study the effect of multipath coherent crosstalk due to optical network nodes on DPSK signals," IEEE Photon. Technol. Lett., vol. 21, no. 20, pp. 1499-1501, Oct. 2009.

[31] R. C. Emerson, "First probability densities for receivers with square law detectors," J. Appl. Phys, vol. 24, no. 9, pp. 1168-1176, Sep. 1953.

[32] G. Bosco and P. Poggiolini, "The impact of receiver imperfections on the performance of optical direct-detection DPSK," J. Lightw. Technol., vol. 23, no. 2, pp. 842-848, Feb. 2005.

[33] E. Forestieri, "Evaluating the error probability in lightwave systems with chromatic dispersion, arbitrary pulse shape and pre-and postdetection filtering," J. Lightw. Technol., vol. 18, no. 11, pp. 1493-1503, Nov. 2000.

[34] E. Forestieri and M. Secondini, "On the error probability evaluation in lightwave systems with optical amplification," J. Lightw. Technol., vol. 27, no. 6, pp. 706-717, Mar. 2009.

[35] E. L. Goldstein, L. Eskildsen, C. Lin, and Y. Silberberg, "Polarization statistics of crosstalk-induced noise in transparent lightwave networks," IEEE Photon. Technol. Lett., vol. 7, no. 11, pp. 1345-1347, Nov. 1995

[36] F. J. Scire, "A probability density function theorem for the modulo $y$ values of the sum of two statistically independent processes," Proc. IEEE, pp. 204-205, Feb. 1968

[37] L. E. Miller and J. S. Lee, "The probability density function for the output of an analog cross-correlator with correlated bandpass inputs," IEEE Trans. Inf. Theory, vol. IT-20, no. 4, pp. 433-440, Jul. 1974.

[38] A. M. Mathai and S. B. Prevost, Quadratic Forms in Random Variables. New York: Marcel Dekker, 1992, pp. 217-221.

[39] B. Saleh, Photoelectron Statistics. Berlin, Heidelberg: SpringerVerlag, 1978.

[40] T. Kamalakis and T. Sphicopoulos, "Asymptotic behaviour of in-band crosstalk noise in WDM networks," IEEE Photon. Technol. Lett., vol. 15 , no. 3, pp. 476-478, Mar. 2003.

[41] I. T. Monroy, E. Tangdiongga, and H. de Waardt, "Performance of optically preamplified receivers in WDM systems disturbed by interferometric crosstalk," Photonic Netw. Commun., vol. 1, no. 4, pp. 313-322, 1999.

[42] W. H. Press, S. A. Teukolsky, W. T. Vetterling, and B. P. Flannery, Numerical Recipes in $\mathrm{C}-$ The Art of Scientific Computing. Cambridge: Cambridge Univ. Press, 1992.

[43] G. Einarsson, Principles of Lightwave Communications. Chichester, U.K.: Wiley, 1996.

[44] C. W. Helstrom, "Computing the distribution of sums of random sine waves and of Rayleigh-distributed random variables by saddle-point integration," IEEE Trans. Commun., vol. 45, no. 11, pp. 1487-1494, Nov. 1997.

[45] J. J. O. Pires and L. G. C. Cancela, "On the probability density function of an optical DPSK signal in the presence of intrachannel crosstalk and ASE noise," J. Opt. Commun., vol. 28, no. 3, pp. 229-233, Oct. 2007.

[46] K.-P. Ho, Phase-Modulated Optical Communication Systems. New York: Springer, 2005

[47] I. Gradshteyn and I. Ryzhic, Table of Integrals, Series, and Products. Orlando, FL: Academic, 2000.

João J. O. Pires received the Ph.D. degree in electrical and computer engineering from the Technical University of Lisbon, Portugal, in 1993.

$\mathrm{He}$ is currently an Assistant Professor at Instituto Superior Técnico, Technical University of Lisbon. From 1991 to 1999 he worked on a number of European-funded projects, including RACE and ACTS projects. He is the author or coauthor of more than 60 papers in international journals and conference proceedings. At present his research interests are mainly in the area of optical transport networks and optical broadband access networks.

Luís G. C. Cancela received the Licenciatura and the M.Sc. degrees both in electrical and computer engineering from the Technical University of Lisbon, Portugal, in 1993 and 1998, respectively. He also received the Ph.D. degree in telecommunications from Instituto Superior das Ciências do Trabalho e da Empresa (ISCTE), Lisbon, Portugal in 2008.

Currently, he is a Researcher at Instituto de Telecomunicações in Lisbon and an Assistant Professor at ISCTE. His research interests include the study of optical crosstalk in fiber-optic communication networks. 